INFLAMMATORY BOWEL DISEASE

\title{
Risk of haematopoietic cancer in patients with inflammatory bowel disease
}

\author{
J Askling, L Brandt, A Lapidus, P Karlén, M Bïörkholm, R Löfberg, A Ekbom
}

Gut 2005;54:617-622. doi: 10.1136/gut.2004.051771

See end of article for authors' affiliations

....................

Correspondence to: Dr J Askling, Clinical Epidemiology Unit M9:01, Karolinska Hospital, SE-171 76 Stockholm, Sweden; johan.askling@ medks.ki.se

Revised version received 5 July 2004

Accepted for publication

17 August 2004

\begin{abstract}
Background and aims: Several chronic inflammatory conditions are associated with an increased risk of lymphoma. Whether this applies to inflammatory bowel disease (IBD) is still unclear but of paramount interest, particularly in the safety evaluation of newer immunosuppressive drugs. Reports also indicate a possible increase in the risk of leukaemia in IBD. We therefore assessed the risk of haematopoietic cancers in a large cohort of patients with IBD.

Subjects and methods: We performed a population based cohort study using prospectively recorded data, including 47679 Swedish patients with Crohn's disease (CD) or ulcerative colitis (UC) assembled from regional cohorts of IBD from 1955 to $1990(n=8028)$ and from the Inpatient Register of 1964-2000 $(n=45060)$, with follow up until 2001. Relative risks were expressed as standardised incidence ratios (SIR).

Results: Overall, we observed 264 haematopoietic cancers during follow up, which corresponded to a borderline significant $20 \%$ increased risk in both UC and CD. In UC, lymphomas occurred as expected (SIR $1.0, n=87$ ) but myeloid leukaemia occurred significantly more often than expected (SIR 1.8, $n=32$ ). In $C D$, there was a borderline significant increased lymphoma risk (SIR 1.3, $n=65$ ), essentially confined to the first years of follow up. Proxy markers of disease activity had little impact on lymphoma risk.

Conclusion: On average, patients with IBD have a marginally increased risk of haematopoietic cancer. In UC, this is accounted for by an excess of myeloid leukaemia. In CD, a modest short term increase in the risk of lymphoma of unknown significance cannot be excluded but any long term risk increase seems unlikely.
\end{abstract}

$\mathrm{D}$ uring the past few years, the risk of haematopoietic cancer in patients with inflammatory bowel disease (IBD), in particular Crohn's disease (CD), has been a growing concern (summarised in table 1). ${ }^{1-17}$ Controversy exists as to whether the underlying risks of lymphoma or leukaemia are increased and, if so, whether they are related to IBD or its treatment. Following the introduction of tumour necrosis factor (TNF) inhibitors in the treatment of $\mathrm{CD}$, subsequent reports have indicated an excess of malignant lymphomas among treated patients ${ }^{18} 19$ and raised fears of an iatrogenic lymphoma risk. However, these reports have also highlighted the lack of robust data on the expected occurrence of malignant lymphomas in TNF naïve (but otherwise treated) patients with IBD. ${ }^{20-23}$ In other inflammatory disorders, such as rheumatoid arthritis, there is a markedly increased lymphoma risk, ${ }^{24} 25$ but our knowledge of the mechanism(s) underlying inflammation associated lymphomas is incomplete.

In IBD, the conclusion from existing studies is that the lymphoma risk remains to be properly assessed (table 1): low numbers of expected lymphomas (from 0 to 10 cases, mean 3 cases) have lead to uncertain risk estimates. Likewise, the method of patient recruitment (referral, hospital, population based) may have affected the results. With follow up times shorter than a few years and failure to assess risk by time since diagnosis of IBD, long term risks may have gone undetected. Time trends over calendar periods have not been assessed, despite an increasing use of immunosuppressive treatment over the past decade(s). Also, clinical decision making should be based on cumulative incidence rather than the exclusively reported relative risks. Bone marrow toxicity of several immunosuppressive agents, azathioprine included, warrants a proper assessment of the risk of (myeloid) leukaemia in IBD, an excess of which has been described. $^{2}{ }^{26-28}$
In this study, we therefore assessed overall and time specific risks of haematopoietic cancers in the hitherto largest cohort of patients with IBD assembled in different but population based settings and followed for cancer occurrence for up to 40 years.

\section{SUBJECTS AND METHODS Study populations}

Patients with IBD were identified from four population based data sources that have been described in detail elsewhere. ${ }^{29-32}$

\section{(1) The Uppsala cohort of IBD}

Established in 1990 using prospectively recorded medical data, the cohort includes incident and prevalent cases in the Uppsala-Örebro health care region, 1965-1983..$^{30}$ Cases were identified through several routes, including histopathological examinations or surgically removed tissue and hospitalisations. The medical charts of all individuals were reviewed. In total, 2509 incident and 613 prevalent cases of ulcerative colitis (UC) and 1469 incident and 187 prevalent cases of CD were identified. For all individuals, information on national registration number (a 10 digit number unique to each Swedish resident ${ }^{33}$ ), date of diagnosis, and extent of inflammation was recorded.

(2) The Stockholm County cohorts of IBD

These cohorts include all known individuals with confirmed UC 1954-1984 or CD 1954-1990 in Stockholm County. ${ }^{71}$ Cases were identified through manual scrutiny of files from the departments of internal medicine, surgery, paediatrics, and infectious diseases in Stockholm, and through

Abbreviations: IBD, inflammatory bowel disease; $C D, C$ rohn's disease; UC, ulcerative colitis; SIR, standardised incidence ratio; TNF, tumour necrosis factor; ICD, International Classification of Diseases 
Table 1 Studies of lymphoma risk in inflammatory bowel disease (IBD)

\begin{tabular}{|c|c|c|c|c|}
\hline Author, year & IBD & No of subjects & Lymphomas & Relative risk $(95 \% \mathrm{CI})$ \\
\hline Lewis' 2001 & $C D$ & 6605 & 7 & $1.6(0.6-3.3)$ \\
\hline Arseneau $^{2} 2001$ & $C D$ & 5426 & 21 & $2.0(1.3-3.1)$ \\
\hline Bernstein $^{3} 2001$ & $C D$ & 2857 & 9 & $2.4(1.2-5.0)$ \\
\hline Mellemkjaer $^{37} 2000$ & $C D$ & 2645 & 6 & $1.4(0.6-1.3)$ \\
\hline Palli $^{38} 1998$ & $C D$ & 231 & 1 & 10 \\
\hline Loftus $^{6} 2000$ & $C D$ & 216 & 4 & $2.4(0.1-13)$ \\
\hline Persson 71994 & $C D$ & 1251 & 4 & $1.3(0.4-3.4)$ \\
\hline Munkholm ${ }^{8} 1993$ & $C D$ & 373 & 0 & 0.0 \\
\hline Ekbom $^{12} 1991$ & $C D$ & 1655 & 1 & $0.4(0.0-2.4)$ \\
\hline$G^{\prime} y e^{10} 1980$ & $C D$ & 513 & 1 & 1.0 \\
\hline Lewis' 2001 & UC & 10391 & 11 & $1.1(0.5-2.2)$ \\
\hline Bernstein $^{3} 2001$ & UC & 2672 & 7 & $1.0(0.5-2.2)$ \\
\hline Palli $^{5} 2000$ & UC & 689 & 6 & 4.2 \\
\hline Loftus ${ }^{6} 2000$ & UC & 238 & 0 & $0.0(0.0-6.4)$ \\
\hline Karlén ${ }^{11} 1999$ & UC & 1547 & 3 & $1.2(0.3-2.5)$ \\
\hline Mellemkjaer ${ }^{4} 1995$ & UC & 5546 & 7 & $1.4(0.5-1.3)$ \\
\hline Ekbom ${ }^{12} 1991$ & UC & 3121 & 8 & $1.2(0.5-2.4)$ \\
\hline Madjlessi $^{28} 1986$ & UC & 1248 & 3 & $3.4(0.7-10)$ \\
\hline Prior 1982 & UC & 676 & 3 & 2.0 \\
\hline Farrell $^{14} 2000$ & NS & 782 & 4 & $31(2.0-64)$ \\
\hline Masel $^{15} 2000$ & NS & 4791 & 5 & 6.7 \\
\hline Connell ${ }^{16} 1994$ & NS & 755 & 0 & 0.0 \\
\hline Greenstein $^{17} 1985$ & NS & 1961 & 8 & 6.1 \\
\hline All studies above & & & 122 & 1.8 \\
\hline
\end{tabular}

hospitalisations. In total, 1573 individuals with UC and 1936 with CD were identified. For each individual, information on national registration number, date of diagnosis, and extent was collected.

\section{(3) The Stockholm pancolitis register}

This cohort encompasses all individuals with pancolitic UC of more than eight years' duration at the gastroenterology unit at South Hospital in Stockholm County 1973-1996. ${ }^{32}$ For this study, information on national identification number and date of diagnosis was retrieved for 143 individuals.

\section{(4) The inpatient register}

The population based Swedish inpatient register contains individual information on inpatient care countywise since 1964 and nationwide since $1987 .^{34}$ For every hospital discharge, information on discharge diagnoses and surgical procedures is recorded according to the International

\begin{tabular}{|c|c|c|c|c|}
\hline & \multicolumn{2}{|c|}{ Ulcerative colitis } & \multicolumn{2}{|c|}{ Crohn's disease } \\
\hline & $\begin{array}{l}\text { Regional } \\
\text { cohorts }\end{array}$ & $\begin{array}{l}\text { Inpatient } \\
\text { register }\end{array}$ & $\begin{array}{l}\text { Regional } \\
\text { cohorts }\end{array}$ & $\begin{array}{l}\text { Inpatient } \\
\text { register }\end{array}$ \\
\hline \multicolumn{5}{|l|}{ Inpatient register } \\
\hline No & 1523 & - & 1096 & - \\
\hline Yes & 2944 & 26036 & 2465 & 19024 \\
\hline \multicolumn{5}{|l|}{ Regional cohort } \\
\hline No & - & 23092 & - & 16559 \\
\hline Yes & 4467 & 2944 & 3561 & 2465 \\
\hline \multicolumn{5}{|l|}{ Sex } \\
\hline Male & 2476 & 14137 & 1689 & 8908 \\
\hline Female & 1991 & 11899 & 1872 & 10116 \\
\hline \multicolumn{5}{|l|}{ Age at entry (y) } \\
\hline $0-39$ & 2177 & 8792 & 2025 & 8398 \\
\hline $40-59$ & 1800 & 11451 & 1267 & 7806 \\
\hline $60+$ & 490 & 5793 & 269 & 2820 \\
\hline \multicolumn{5}{|l|}{ Year of entry } \\
\hline-1969 & 1575 & 1327 & 1053 & 929 \\
\hline $1970-1979$ & 2008 & 5037 & 1480 & 4176 \\
\hline $1980-1989$ & 884 & 9005 & 1028 & 6475 \\
\hline 1990-1999 & - & 10667 & - & 7444 \\
\hline \multicolumn{5}{|l|}{ Extent of inflammation } \\
\hline Proctitis/terminal ileitis & 1492 & - & 1314 & - \\
\hline Left sided/ileocolic & 1111 & - & 1095 & - \\
\hline Pancolitis/colon Crohn & 1580 & - & 951 & - \\
\hline Not specified & 284 & - & 201 & - \\
\hline \multicolumn{5}{|l|}{ Accrued follow up (y) } \\
\hline $1-4$ & 3696 & 25454 & 3202 & 18734 \\
\hline $5-9$ & 3918 & 19913 & 3343 & 15235 \\
\hline $10-19$ & 4026 & 14021 & 3360 & 11194 \\
\hline$\geqslant 20$ & 3181 & 5390 & 2332 & 4777 \\
\hline
\end{tabular}


Classification of Diseases $\left(\mathrm{ICD}^{35}\right)$, versions 7-10. We identified all patients discharged with a diagnosis of IBD between 1 January 1964 and 31 December 2000. ICD codes used for CD were: (ICD7) 572.00, 572.09, (ICD8) 563.00, (ICD9) 555, (ICD10) K50; and for UC: (ICD7) 572.20, 572.21 (ICD8) 563.10, 569.02 (ICD9) 556 (ICD10) K51. For each individual, information on diagnosis, date of first discharge with IBD, age, sex, and national registration number was recorded. For individuals discharged with both CD and UC, the first date of discharge but the last type of recorded IBD was used. In total, 50105 individuals with IBD were identified.

For individuals found in any regional cohort (cohorts 1-3) and in the inpatient register (cohort 4), the mean time between date of diagnosis, as stated in either regional cohort, and the date of first discharge in the inpatient register was 4.4 years (median 0.5 years); $96 \%$ of these were registered with the same type of IBD in both the regional cohort and in the inpatient register. Because of the overlap between the regional and inpatient register cohorts, and after exclusion of patients with follow up times shorter than one year (see below), the analytical cohort comprised a total of 47679 individuals (table 2).

\section{Follow up}

To identify incident haematopoietic cancers, the cohorts were linked with the Swedish Cancer Register 1958-2000. For each cancer site (tables 3,4), information on date of diagnosis was collected. To collect information on emigration and vital status, the cohorts were also linked with the Register of Total Population 1969-2000 and with the Cause of Death Register 1952-2000. For patients identified in any regional cohort, follow up started at the registered date of diagnosis of IBD. For patients exclusively identified in the inpatient register, follow up started at the time of first discharge listing IBD. Follow up ended at the first of: date of emigration, date of death, and end of the study period (31 December 2000). To assess whether patients never hospitalised for their IBD differed from ever hospitalised patients, we performed separate analyses of the regional cohorts in which patients were also censored at first hospitalisation for IBD.

\section{Statistics}

We used standardised incidence ratios (SIR, the ratio of the observed and expected number of cancers) as measures of relative risk. Expected numbers were calculated by multiplying sex, age, and calendar period specific person years of follow up with corresponding rates from the entire Swedish population; 95\% confidence intervals (CI) were calculated assuming a Poisson distribution of the observed cases. ${ }^{36}$ To avoid bias from misdiagnosis and selection, we excluded observed and expected cancers during the first year of follow up from all estimates of relative risk. Adjusted relative risks were estimated using Poisson regression with the expected number of cancers as offset. Because of the striking similarity in relative risks between the regional and inpatient cohorts, we combined these into one when modelling the SIR using Poisson regression. (Because of overlap, the sum of observed cancers in either cohort exceeded the total number of cancers in the two cohorts combined.) Estimates of cumulative incidence were calculated using the Kaplan-Meier method in SAS. The study was approved by the ethics committee at Karolinska Institutet.

\section{RESULTS}

\section{Ulcerative colitis}

The relative risk of haematopoietic cancer (45 and 147 cancers in the regional and inpatient cohorts, respectively, 165 in total) was increased $10-20 \%$ in UC (table 3), corresponding to an excess of 0.07 haematopoietic cancers per 1000 person years, but this did not reach statistical significance. The relative risk of malignant lymphomas ( $\mathrm{n}=87$ in total) was close to 1.0 in either cohort, and varied only modestly with sex, calendar period, and time of follow up (tables 3, 5). Likewise, there was little to suggest elevated risks for multiple myeloma or lymphatic leukaemia (table 3 ). Myeloid leukaemia $(n=32)$ occurred more often than expected, with relative risks between 1.5 and 2.9 in the two cohorts (table 3). Multivariate modelling of the relative risk of myeloid leukaemia (acute and chronic) revealed a nonsignificant tendency towards higher relative risks among men ( $v$ women) and during the 1990s ( $v$ 1980-1989), and that the excess number of cases occurred after more than five years of follow up (table 6).

\section{Crohn's disease}

The relative risk of haematopoietic cancer (27 and 98 in the regional and inpatient cohorts, respectively, 99 in total) in CD was increased by $15-20 \%$ (significantly so only in the inpatient register cohort (table 4)), corresponding to an excess of 0.06 cancers per 1000 person years. The relative risk

Table 3 Cancer risk among 27559 Swedish patients with ulcerative colitis, identified in two regional and population based cohorts and/or in the nationwide and population based register of inpatient care

\begin{tabular}{|c|c|c|c|c|c|c|}
\hline \multirow[b]{3}{*}{ Cancer type } & \multicolumn{6}{|c|}{ Ulcerative colitis ( $n=27$ 559*) } \\
\hline & \multicolumn{3}{|c|}{ Regional cohorts $(n=4467)$} & \multicolumn{3}{|c|}{ Inpatient register ( $n=26036$ ) } \\
\hline & 0 & E & SIR $(95 \%$ Cl) & 0 & E & SIR $(95 \% \mathrm{Cl})$ \\
\hline All solid or haematopoietic sites (140-209) & 635 & 502.5 & $1.26(1.2-1.4)$ & 2124 & 1739.9 & $1.22(1.2-1.3)$ \\
\hline All haematopoietic cancers $(200-209)$ & 45 & 38.4 & $1.17(0.9-1.6)$ & 147 & 132.6 & $1.11(0.9-1.3)$ \\
\hline Malignant lymphomas including CLL $(200-202,204.1)$ & 19 & 22.7 & $0.84(0.5-1.3)$ & 78 & 79.6 & $0.98(0.8-1.2)$ \\
\hline Non-Hodgkin lymphomas $(200,202)$ & 17 & 15.5 & $1.10(0.6-1.8)$ & 63 & 55.6 & $1.13(0.9-1.4)$ \\
\hline Hodgkin's lymphoma (201) & 1 & 2.6 & $0.39(0.01-2.2)$ & 8 & 7.7 & $1.03(0.4-2.0)$ \\
\hline CLL (204.1) & 1 & 4.6 & $0.22(0.01-1.2)$ & 7 & 16.3 & $0.43(0.2-0.9)$ \\
\hline Multiple myeloma (203) & 6 & 6.7 & $0.90(0.3-2.0)$ & 29 & 23.0 & $1.26(0.8-1.8)$ \\
\hline All leukaemias excluding CLL $(204-207,209)$ & 17 & 7.3 & $2.32(1.4-3.7)$ & 35 & 24.8 & $1.13(0.9-1.4)$ \\
\hline Acute lymphatic leukaemia $(204.0,204.9)$ & 1 & 0.7 & $1.40(0.04-7.8)$ & 2 & 2.4 & $0.84(0.1-3.0)$ \\
\hline Acute myeloid leukaemia $(205.0,205.9)$ & 9 & 3.6 & $2.53(1.2-4.8)$ & 19 & 12.4 & $1.53(0.9-2.4)$ \\
\hline Chronic myeloid leukaemia (205.1) & 4 & 1.4 & $2.86(0.8-7.3)$ & 10 & 4.3 & $2.35(1.1-4.3)$ \\
\hline Other leukaemias $(206.0-1,207.0-3,207.9,209)$ & 3 & 1.6 & $1.82(0.4-5.3)$ & 4 & 5.7 & $0.70(0.2-1.8)$ \\
\hline Polycytaemia vera (209) & 3 & 1.6 & $1.86(0.4-5.4)$ & 5 & 5.2 & $0.96(0.3-2.2)$ \\
\hline
\end{tabular}

*The sum of 4467 and 26036 exceeded 27559 because of overlap between the cohorts.

CLL, chronic lymphocytic leukaemia. Cancers coded according to ICD-7.

Follow up from 1969 until 2001. Observed (O) and expected (E) number of cases, and standardised incidence ratio (SIR) with $95 \%$ confidence interval (95\% CI). First year of follow up excluded. 
Table 4 Cancer risk among 20120 Swedish patients with Crohn's disease, identified in two regional and population based cohorts and/or in the nationwide and population based register of inpatient care

\begin{tabular}{|c|c|c|c|c|c|c|}
\hline \multirow[b]{3}{*}{ Cancer type } & \multicolumn{6}{|c|}{ Crohn's disease $(n=20120 *)$} \\
\hline & \multicolumn{3}{|c|}{ Regional cohorts ( $n=3561$ ) } & \multicolumn{3}{|c|}{ Inpatient register ( $n=19024$ ) } \\
\hline & 0 & E & SIR $(95 \% \mathrm{CI})$ & 0 & E & SIR $(95 \% \mathrm{CI})$ \\
\hline All solid or haematopoietic sites (140-209) & 318 & 309.1 & $1.03(0.9-1.2)$ & 1188 & 1069.3 & $1.11(1.0-1.2)$ \\
\hline All hematopoietic cancers $(200-209)$ & 27 & 23.4 & $1.15(0.8-1.7)$ & 98 & 80.1 & $1.21(1.0-1.5)$ \\
\hline Malignant lymphomas including CLL $(200-202,204.1)$ & 18 & 14.1 & $1.28(0.8-2.0)$ & 65 & 49.1 & $1.32(1.0-1.7)$ \\
\hline Non-Hodgkin lymphomas $(200,202)$ & 14 & 9.68 & $1.45(0.8-2.4)$ & 53 & 34.2 & $1.55(1.2-2.0)$ \\
\hline Hodgkin's lymphoma (201) & 2 & 1.8 & $1.11(0.1-4.0)$ & 6 & 5.8 & $1.03(0.4-2.2)$ \\
\hline CLL (204.1) & 2 & 2.6 & $0.78(0.09-2.8)$ & 6 & 9.0 & $0.66(0.2-1.4)$ \\
\hline Multiple myeloma (203) & 3 & 3.8 & $0.79(0.2-2.3)$ & 10 & 13.1 & $0.76(0.4-1.4)$ \\
\hline All leukaemias excluding CLL $(204-207,209)$ & 5 & 4.6 & $1.09(0.4-2.5)$ & 21 & 15.7 & $1.34(0.8-2.0)$ \\
\hline Acute lymphatic leukaemia $(204.0,204.9)$ & 1 & 0.5 & $2.09(0.05-11.6)$ & 1 & 1.7 & $0.60(0.02-3.4)$ \\
\hline Acute myeloid leukaemia $(205.0,205.9)$ & 2 & 2.2 & $0.89(0.1-3.2)$ & 4 & 7.8 & $0.51(0.1-1.3)$ \\
\hline Chronic myeloid leukaemia (205.1) & 1 & 0.9 & $1.10(0.03-6.1)$ & 8 & 2.9 & $2.80(1.2-5.5)$ \\
\hline Other leukaemias $(206.0-1,207.0-3,207.9,209)$ & 1 & 1.0 & $1.04(0.03-5.8)$ & 8 & 3.4 & $2.38(1.0-4.7)$ \\
\hline 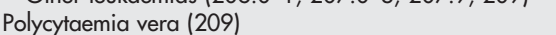 & 1 & 1.0 & $1.04(0.03-5.8)$ & 2 & 3.1 & $0.65(0.1-2.3)$ \\
\hline
\end{tabular}

*The sum of 3651 and 19024 exceeded 20120 because of overlap between the cohorts.

$\mathrm{CLL}$, chronic lymphocytic leukaemia. Cancers coded according to ICD-7.

Follow up from 1969 until 2001. Observed (O) and expected (E) number of cases, and standardised incidence ratio (SIR) with 95\% confidence interval (95\% CI).

First year of follow up excluded.

of malignant lymphoma $(n=65)$ was increased by $30 \%$ in both cohorts but reached formal significance only in the inpatient register cohort (table 4). Much of this excess occurred during the first few years of follow up (table 5). The relative risk of non-Hodgkin lymphomas was increased by $50 \%$ in both cohorts whereas chronic lymphocytic leukaemia occurred less often than expected (table 4). The relative risk of other leukaemias was not elevated in the regional cohort (table 4). In the inpatient register cohort, there was an increased occurrence of chronic myeloid leukaemia and of unspecified leukaemia (table 4). Multivariate modelling of myeloid leukaemia in CD revealed little variation with sex, age, or time (table 6).

\section{Lymphoma risk in relation to extent of disease, hospitalisation, and surgery}

The lymphoma risk in patients for whom we had information on extent of inflammation at time of diagnosis of IBD (4441 UC and 3587 CD) was similar to that of the rest of the patients, without any clear variation in risk with extent of inflammation (data not shown). For both UC and CD, overall and site specific cancer risks in non-hospitalised patients were similar to those of the entire regional cohort, except that in CD; the relative risk (up until the first hospitalisation for CD) was not increased ( SIR $=0.82(95 \%$ CI $0.2-2.1) ; \mathrm{n}=4)$. Neither the number of hospitalisations listing IBD nor a history of intestinal surgery modified the lymphoma risk in $\mathrm{UC}$ or $\mathrm{CD}$.

\section{Cumulative incidence of lymphoma and lymphoma subtype}

The absolute risk of malignant lymphomas after three decades was $1.1 \%$ and $0.7 \%$ for patients with UC and CD, respectively. These absolute risks were similar in the regional and inpatient register cohorts. A manual review of the registered subtype of 103 lymphomas that were reported in 1993 (when information on subtype was included in the Swedish cancer register) or later suggested that the distributions of lymphoma subtypes were similar in CD and in UC, and that the proportion of $\mathrm{T}$ cell lymphomas was approximately $10 \%$.

Table 5 Malignant lymphomas 1969-2001 in a combined population based cohort of 47679 Swedish patients with inflammatory bowel disease

\begin{tabular}{|c|c|c|c|c|c|c|c|c|c|c|}
\hline & \multicolumn{5}{|c|}{ Ulcerative colitis } & \multicolumn{5}{|c|}{ Crohn's disease } \\
\hline & 0 & E & $\operatorname{SIR}(95 \% \mathrm{Cl})$ & RR & $p$ Value & 0 & E & SIR $(95 \% \mathrm{Cl})$ & RR & p Value \\
\hline Overall & 87 & 84 & $1.0(0.8-1.3)$ & - & - & 65 & 50 & $1.3(1.0-1.6)$ & - & - \\
\hline \multicolumn{11}{|l|}{ Sex } \\
\hline Male & 50 & 53 & $0.9(0.7-1.2)$ & 1 (ref) & 0.29 & 42 & 29 & $1.5(1.1-2.0)$ & 1 (ref.) & 0.20 \\
\hline Female & 37 & 31 & $1.2(0.8-1.6)$ & $1.3(0.8-1.9)$ & & 23 & 22 & $1.1(0.7-1.6)$ & $0.7(0.4-1.2)$ & \\
\hline \multicolumn{11}{|l|}{ Cohort of origin } \\
\hline Any regional cohort & 19 & 23 & $0.8(0.5-1.3)$ & $0.8(0.5-1.5)$ & 0.56 & 18 & 14 & $1.3(0.8-2.0)$ & $1.0(0.6-2.0)$ & 0.76 \\
\hline Inpatient register only & 68 & 62 & $1.1(0.9-1.4)$ & 1 (ref) & & 47 & 36 & $1.3(1.0-1.7)$ & 1 (ref.) & \\
\hline \multicolumn{11}{|l|}{ Calendar period at follow up } \\
\hline-1979 & 4 & 5.8 & $0.7(0.2-1.8)$ & 1 (ref) & 0.63 & 5 & 3.1 & $1.6(0.5-3.8)$ & 1 (ref.) & 0.34 \\
\hline 1980-1989 & 24 & 22 & $1.1(0.7-1.6)$ & $1.6(0.5-4.5)$ & & 11 & 12 & $0.9(0.5-1.6)$ & $0.6(0.2-1.8)$ & \\
\hline $1990-2001$ & 59 & 57 & $1.0(0.8-1.3)$ & $1.6(0.5-4.5)$ & & 49 & 35 & $1.4(1.0-1.8)$ & $1.0(0.4-2.6)$ & \\
\hline \multicolumn{11}{|l|}{ Time of follow up (y) } \\
\hline $1-5$ & 36 & 26 & $1.4(1.0-1.9)$ & $1.4(0.8-2.3)$ & 0.09 & 23 & 14 & $1.6(1.0-2.4)$ & $1.1(0.5-2.2)$ & 0.42 \\
\hline $6-10$ & 14 & 19 & $0.7(0.4-1.2)$ & $0.7(0.4-1.4)$ & & 12 & 12 & $1.0(0.5-1.8)$ & $1.0(0.4-1.6)$ & \\
\hline$\geqslant 11$ & 37 & 39 & $1.0(0.7-1.3)$ & 1 (ref) & & 30 & 25 & $1.2(0.8-1.8)$ & 1 (ref.) & \\
\hline
\end{tabular}

First year of follow up excluded.

Observed (O) and expected (E) number of malignant lymphomas, standardised incidence ratio (SIR) with $95 \%$ confidence intervals (95\% CI), and multivariate relative risks (RR) from models including all parameters in table ("ref" denotes reference category).

p values refer to type 3 tests. 
Table 6 Myeloid leukaemia (acute and chronic) 1969-2001 in a combined population based cohort of 47679 Swedish patients with inflammatory bowel disease

\begin{tabular}{|c|c|c|c|c|c|c|c|c|c|c|}
\hline & \multicolumn{5}{|c|}{ Ulcerative colitis } & \multicolumn{5}{|c|}{ Crohn's disease } \\
\hline & 0 & E & $\operatorname{SIR}(95 \% \mathrm{CI})$ & RR & p Value & 0 & E & $\operatorname{SIR}(95 \% \mathrm{CI})$ & RR & p Value \\
\hline Overall & 32 & 18 & $1.8(1.2-2.6)$ & - & - & 13 & 11 & $1.2(0.6-2.0)$ & - & - \\
\hline \multicolumn{11}{|l|}{ Sex } \\
\hline Male & 23 & 10 & $2.2(1.4-3.3)$ & 1 (ref.) & 0.13 & 5 & 5.6 & $0.9(0.3-2.1)$ & 1 (ref.) & 0.34 \\
\hline Female & 9 & 7.3 & $1(0.6-2.3)$ & $0.6(0.3-1.2)$ & & 8 & 5.3 & $1.5(0.7-3.0)$ & $1.7(0.6-5.2)$ & \\
\hline \multicolumn{11}{|l|}{ Cohort of origin } \\
\hline Any regional cohort & 13 & 5.0 & $2.6(1.4-4.5)$ & $2.2(0.9-5.0)$ & 0.07 & 3 & 3.2 & $0.9(0.2-2.8)$ & $0.9(0.2-3.6)$ & 1 \\
\hline Inpatient register only & 19 & 13 & $1.5(0.9-2.3)$ & 1 (ref.) & & 10 & 7.8 & $1.3(0.6-2.4)$ & 1 (ref.) & \\
\hline \multicolumn{11}{|l|}{ Calendar period at follow up } \\
\hline-1979 & 0 & 1.5 & $0.0(0.0-2.4)$ & & & 1 & 0.9 & $1.1(0.0-6.4)$ & 1 (ref.) & 0.46 \\
\hline 1980-1989 & 9 & 4.9 & $1.8(0.8-3.5)$ & 1 (ref.)* & 0.15 & 4 & 2.9 & $1.4(0.4-3.6)$ & $1.2(0.1-11)$ & \\
\hline $1990-2001$ & 23 & 11 & $2.1(1.3-3.1)$ & $1.8(0.8-4.3)$ & & 8 & 7.2 & $1.1(0.5-2.2)$ & $1.1(0.1-9.6)$ & \\
\hline \multicolumn{11}{|l|}{ Time of follow up (y) } \\
\hline $1-5$ & 6 & 5.6 & $1.1(0.4-2.3)$ & $0.9(0.3-2.5)$ & 0.19 & 4 & 3.2 & $1.2(0.3-3.2)$ & $1.5(0.3-)$ & 0.47 \\
\hline $6-10$ & 11 & 4.1 & $2.7(1.3-4.8)$ & $1.9(0.8-4.6)$ & & 5 & 2.6 & $1.9(0.6-4.5)$ & $2.4(0.6-9.5)$ & \\
\hline$\geqslant 11$ & 15 & 7.9 & $1.9(1.1-3.1)$ & 1 (ref.) & & 4 & 5.1 & $0.8(0.2-2.0)$ & 1 (ref.) & \\
\hline
\end{tabular}

*-1979 and 1980-1989 were combined into one category.

First year of follow up excluded.

Observed (O) and expected (E) number of myeloid leukaemia, standardised incidence ratio (SIR) with $95 \%$ confidence intervals $(95 \% \mathrm{CI})$, and multivariate relative risks (RR) from models including all parameters in table ("ref" denotes reference category).

$p$ values refer to type 3 tests.

\section{DISCUSSION}

The results of our population based, partly nationwide cohort study of nearly 50000 patients with IBD suggest that the overall risk of haematopoietic cancer in IBD may be increased, but only modestly so, and differently in UC and in CD. In UC, there was a markedly increased occurrence of myeloid leukaemia but no increased occurrence of lymphomas. In CD, the overall lymphoma risk was modestly elevated but confined to the first few years of follow up with little to indicate a long term increase in risk.

Our finding of no increased lymphoma risk in UC is in accordance with previous studies in which relative risks typically have been between 1.0 and 1.5 (crude average approximately 1.4). ${ }^{13-6} 9^{11-13}$ It is therefore fair to conclude that ulcerative colitis is not linked to an increased occurrence of malignant lymphomas. Clinically, this means that reports of excess numbers of lymphomas in trials of new therapeutic regimens in UC should raise concern. ${ }^{19}$ Biologically, this suggests that inflammation in UC is less lymphomagenic than, for example, in rheumatoid arthritis. ${ }^{24}$

The slight increased lymphoma risk in CD was lower than that in the most recent and so far largest studies, ${ }^{1-3}$ and lower than the average of all previous studies combined (crude average relative risk 1.8). ${ }^{1-3} 6-810123738$ In our study, there was little to support a time dependent lymphoma risk or any heterogeneity in risk over proxy makers for disease activity, such as number of hospitalisations and intestinal surgery, but (based on small numbers) non-hospitalised CD patients were not at increased risk of lymphoma. Although we cannot formally exclude the possibility that subsets of patients with CD (characterised by factors other than those measured in this study) may be at increased risk of lymphoma, such subsets, and/or their lymphoma risk, must be small or modest.

In the hitherto largest (entirely register based) study of lymphoma risk in IBD, Lewis et al followed 17000 prevalent cases of IBD for a mean of 3.8 years. ${ }^{1}$ Exposure (IBD) and outcome (lymphoma) were identified in the same register. The relative risk of lymphoma was (non-significantly) 30\% increased in CD and 10\% increased in UC, based on seven and 11 observed cases, respectively, but relative risks by time could not be assessed, and absolute risks were not reported. Arseneau et al also used an entirely register based design, followed patients with IBD over a two year period, and observed a doubled lymphoma/leukaemia (only this combined end point was reported) risk. $^{2}$ Using a similar design, Bernstein et al observed a more than double risk of lymphoma in (men with) CD but no risk increase in UC. ${ }^{3}$ Studies focusing on lymphoma risk following immunosuppressive treatment in IBD have (with one exception ${ }^{14}$ ) found little to suggest a lymphomagenic potential of azathioprine. ${ }^{16}{ }^{39}$ With respect to leukaemia risk in $\mathrm{IBD}$, several studies have indicated conspicuous occurrences of myelodysplastic syndromes/myeloid leukaemia and IBD $^{17} 272840-42$ but large scale studies, including data on risks associated with azathioprine, are lacking. In our study, there was a non-significant tendency towards higher risks for myeloid leukaemia in UC during the 1990s but no such trend was observed for CD. Because of the relatively recent introduction of azathioprine in the treatment of IBD in Sweden (azathioprine and 6-mercaptopurine has been less frequently used in Sweden than in the USA but the use has increased rapidly in the past decade) any causal association between azathioprine and myeloid leukaemia remains to be established.

While questions such as data validity, selection, and information bias limit inferences from the above described register studies on haematopoietic cancer in $\mathrm{IBD}^{1-3}$ and others using similar designs, ${ }^{47}$ reports based on detailed and manually scrutinised cohorts have often yielded imprecise estimates. $^{57^{9-12} 38}$ The main strength of the present study was the combination of large, detailed, population based, regional cohorts with a population based, larger, register based but less detailed (nationwide) cohort of patients with IBD. The study population not only provided good precision even for rare outcomes but also the means of assessing the consistency between the two types of cohorts. This high degree of consistency suggests that the observed risks represent valid measures in unselected populations of IBD. In the regional cohorts, the follow up of which was considerable extended compared with previous reports, the diagnoses of IBD were subject to manual scrutiny of medical files and thus were highly valid. ${ }^{71}{ }^{12}$ Reassuringly, among patients appearing in both the regional and inpatient cohorts, there was a high concordance $(96 \%)$ for the type of IBD reported in each cohort. Recall bias and information bias were both minimised by the use of prospectively and independently recorded data on exposure and outcome, the latter identified in a nationwide register. The study period (up to four decades) provided 
a means of assessing variations in risk with time since diagnosis of IBD and, importantly, trends over successive decades. The increased risk of malignant lymphomas in CD during the first few years of follow up is likely to reflect misclassification of a malignant lymphoma as IBD or selection bias caused by a diagnosis of IBD for reasons related to an emerging lymphoma. TNF inhibitors have been used in the treatment of CD in Sweden since the late 1990s. Our results thus essentially apply to TNF naïve patients.

Our study has some limitations. Information on extent of IBD at diagnosis was only available for patients identified in the regional cohorts, and we had no information on pharmacotherapy. Instead, we used proxy markers for disease severity (but found no association with lymphoma risk). The lack of uniform reclassification of the observed cancers according to modern techniques and classification criteria precluded a more detailed assessment of subtype specific lymphoma risks, although the proportion of $\mathrm{T}$ cell lymphomas was close to that expected.

In conclusion, patients with IBD have an increased relative risk of haematopoietic cancer, which in absolute terms is small. There is a markedly increased relative risk of myeloid leukaemia in UC. With respect to lymphomas, these do not occur more often than expected in UC. In CD, the relative risk is, if anything, only marginally increased, and displays little variation with factors associated with disease severity. Clinically, this suggests that reports on lymphomas in IBD treated with new drugs should raise concerns. Biologically, this suggests that the mechanisms governing inflammation associated malignant lymphomas appear to exert greater influence in rheumatoid arthritis than in IBD.

\section{ACKNOWLEDGEMENT}

Supported by the Swedish Cancer Society 4537-B02-02XBB, 4755B02-01XAC.

\section{Authors' affiliations}

J Askling, L Brandt, Clinical Epidemiology Unit, Department of Medicine, Karolinska Institutet and Hospital, Stockholm, Sweden A Lapidus, Centre for Gastrointestinal Disease, Ersta Hospital, Stockholm, Sweden

P Karlén, Gastroenterology Unit, Department of Medicine, South Hospital, Karolinska Institutet, Stockholm, Sweden

M Björkholm, Division of Haematology, Department of Medicine, Karolinska Institutet and Hospital, Stockholm, Sweden

R Löfberg, Clinical Epidemiology Unit, Department of Medicine, Karolinska Institutet and Hospital, and IBD Unit, HMQ Sophia Hospital, Stockholm, Sweden

A Ekbom, Clinical Epidemiology Unit, Department of Medicine, Karolinska Institutet and Hospital, Stockholm, Sweden, and Department of Epidemiology, Harvard School of Public Health, Boston, MA, USA

Conflict of interest: None declared.

\section{REFERENCES}

1 Lewis JD, Bilker WB, Brensinger C, et al. Inflammatory bowel disease is not associated with an increased risk of lymphoma. Gastroenterology $2001 ; 121: 1080-7$.

2 Arseneau KO, Stukenborg GJ, Connors AF Jr, et al. The incidence of lymphoid and myeloid malignancies among hospitalized Crohn's disease patients. Inflamm Bowel Dis $2001 ; 7: 106-12$

3 Bernstein CN, Blanchard JF, Kliewer E, et al. Cancer risk in patients with inflammatory bowel disease: a population-based study. Cancer 2001;91:854-62.

4 Mellemkjaer L, Olsen JH, Frisch $M$, et al. Cancer in patients with ulcerative colitis. Int J Cancer 1995;60:330-3.

5 Palli D, Trallori G, Bagnoli S, et al. Hodgkin's disease risk is increased in patients with ulcerative colitis. Gastroenterology 2000;1 19:647-53.

6 Loftus EV, Tremaine WJ, Habermann TM, et al. Risk of lymphoma in inflammatory bowel disease. Am J Gastroenterol 2000;95:2308-12.

7 Persson PG, Karlen P, Bernell O, et al. Crohn's disease and cancer: a population-based cohort study. Gastroenterology 1994;107:1675-9.
8 Munkholm P, Langholz E, Davidsen $M$, et al. Intestinal cancer risk and mortality in patients with Crohn's disease. Gastroenterology 1993; 105:1716-23

9 Prior P, Gyde SN, Macartney JC, et al. Cancer morbidity in ulcerative colitis. Gut 1982;23:490-7.

10 Gyde SN, Prior P, Macartney JC, et al. Malignancy in Crohn's disease. Gut 1980;21:1024-9.

11 Karlén P, Lofberg R, Brostrom O, et al. Increased risk of cancer in ulcerative colitis: a population-based cohort study. Am J Gastroenterol 1999:94:1047-52.

12 Ekbom A, Helmick C, Zack M, et al. Extracolonic malignancies in inflammatory bowel disease. Cancer 1991;67:2015-19.

13 Mir-Madjlessi SH, Farmer RG, Easley KA, et al. Colorectal and extracolonic malignancy in ulcerative colitis. Cancer 1986;58:1569-74.

14 Farrell RJ, Ang Y, Kileen P, et al. Increased incidence of non-Hodgkin's lymphoma in inflammatory bowel disease patients on immunosuppressive therapy but overall risk is low. Gut 2000;47:514-19.

15 Masel S, Hanauer S. Increased asscociation of lymphoma and inflammatory bowel disease. Gastroenterology 2000;118:A119.

16 Connell WR, Kamm MA, Dickson M, et al. Long-term neoplasia risk after azathioprine treatment in inflammatory bowel disease. Lancet 1994;343:1249-52.

17 Greenstein AJ, Gennuso R, Sachar DB, et al. Extraintestinal cancers in inflammatory bowel disease. Cancer 1985;56:2914-21.

18 Brown SL, Greene MH, Gershon SK, et al. Tumor necrosis factor antagonist therapy and lymphoma development: twenty-six cases reported to the Food and Drug Administration. Arthritis Rheum 2002;46:3151-8.

19 Ljung T, Karlen P, Schmidt D, et al. Infliximab in inflammatory bowel disease: clinical outcome in a population based cohort from Stockholm County. Gut 2004;53:849-53

20 Loftus EV Jr, Sandborn WJ. Lymphoma risk in inflammatory bowel disease: influences of referral bias and therapy. Gastroenterology 2001:121:1239-42.

21 Sandborn WJ, Loftus EV. Balancing the risks and benefits of infliximab in the treatment of inflammatory bowel disease. Gut 2004;53:780-2.

22 Bebb JR, Logan RP. Review article: does the use of immunosuppressive therapy in inflammatory bowel disease increase the risk of developing lymphoma? Alimentary Pharmacol Ther 2001;15:1843-9.

23 Aithal GP, Mansfield JC. Review article: the risk of lymphoma associated with inflammatory bowel disease and immunosuppressive treatment. Aliment Pharmacol Ther 2001;15:1101-8.

24 Ekstrom K, Hjalgrim H, Brandt L, et al. Risk of malignant lymphomas in patients with rheumatoid arthritis and in their first-degree relatives. Arthritis Rheum 2003;48:963-70.

25 Baecklund E, Ekbom A, Sparen P, et al. Disease activity and risk of lymphoma in patients with rheumatoid arthritis: nested case-control study. BMJ 1998:317:180-1.

26 Fabry TL, Sachar DB, Janowitz HD. Acute myelogenous leukaemia in patients with ulcerative colitis. J Clin Gastroenterol 1980;2:225-7.

27 Harewood GC, Loftus EV Jr, Tefferi A, et al. Concurrent inflammatory bowel disease and myelodysplastic syndromes. Inflamm Bowel Dis 1999:5:98-103.

28 Mir Madjlessi SH, Farmer RG, Weick JK. Inflammatory bowel disease and leukaemia. A report of seven cases of leukaemia in ulcerative colitis and Crohn's disease and review of the literature. Dig Dis Sci 1986;31:1025-31.

29 Persson PG, Bernell O, Leijonmarck CE, et al. Survival and cause-specific mortality in inflammatory bowel disease: a population-based cohort study. Gastroenterology 1996;110:1339-45.

30 Ekbom A, Helmick C, Zack M, et al. The epidemiology of inflammatory bowel disease: a large, population-based study in Sweden. Gastroenterology 1991; 100:350-8

31 Lapidus A, Bernell O, Hellers G, et al. Incidence of Crohn's disease in Stockholm County 1955-1989. Gut 1997:41:480-6.

32 Lofberg R, Brostrom O, Karlen P, et al. Colonoscopic surveillance in long standing total ulcerative colitis-a 15-year follow-up study. Gastroenterology 1990;99:1021-31.

33 Lunde AS, Lundeborg S, Lettenstrom GS, et al. The person-number systems of Sweden, Norway, Denmark, and Israel. Vital Health Stat 2 1980;2:1-59.

34 Patientregistret 1987-1996 Kvalitet och innehåll. Stockholm: Epidemiologiskt Centrum, Socialstyrelsen, 1998.

35 International Classification of Diseases. Geneva: World Health Organisation, 1955.

36 Breslow NE, Day NE. Statistical methods in cancer research. vol II-The design and analysis of cohort studies. Oxford: Oxford University Press, 1987.

37 Mellemkjaer L, Johansen C, Gridley G, et al. Crohn's disease and cancer risk (Denmark). Cancer Causes Control 2000;11:145-50.

38 Palli D, Trallori G, Saieva C, et al. General and cancer specific mortality of a population based cohort of patients with inflammatory bowel disease: the Florence Study. Gut 1998;42:175-9.

39 Korelitz BI, Mirsky FJ, Fleisher MR, et al. Malignant neoplasms subsequent to treatment of inflammatory bowel disease with 6-mercaptopurine. Am J Gastroenterol 1999;94:3248-53.

40 Caspi O, Polliack A, Klar R, et al. The association of inflammatory bowel disease and leukaemia - coincidence or not? Leuk Lymphoma 1995; 17:255-62.

41 Cohn EM, Pearlstine B. Inflammatory bowel disease and leukaemia. J Clin Gastroenterol 1984;6:33-5.

42 Heizer WD, Peterson JL. Acute myeloblastic leukaemia following prolonged treatment of Crohn's disease with 6-mercaptopurine. Dig Dis Sci 1998;43:1791-3. 